\title{
Effectiveness of flash of light as a distraction technique on level of pain among hospitalized children undergoing IV cannulation at selected hospital, Bangalore.
}

\author{
Mrs Pusparani Loitam \\ Msc Nursing, Padmashree Institute Of Nursing, Bangalore \\ DOI: 10.29322/IJSRP.11.08.2021.p11680 \\ http://dx.doi.org/10.29322/IJSRP.11.08.2021.p11680
}

\section{BACKGROUND}

$\mathrm{P}$ ain is a stressful experience that is considered to be a global health problem, and children are the most vulnerable and under-served population. Intravenous cannulation is a distressing and painful procedure for patients. It causes moderate or severe pain in a substantial number of children.There are numerous pain reduction methods for children undergoing venipuncture, these are not widely used due to the increased time it takes for implementation or their cost and availability in medical offices/hospitals.

Many non-pharmacological techniques such as distraction, guided imagery, virtual reality glasses, cartoon, non-procedural talk and cutaneous stimulation provides coping strategies that may help to reduce pain. In fact distraction is the most common non pharmacological technique utilized by parents and health care professionals to decrease pain among children.

\section{OBJectives}

1. To assess the level of pain after intervention among hospitalized children undergoing I.V cannulation in the experimental group.

2. To assess the level of pain among hospitalized children undergoing I.V cannulation in the control group.

3. To compare the level of pain among hospitalized children undergoing I.V cannulation between experimental and control group.

4. To associate the level of pain among hospitalized children undergoing IV cannulation with their selected background variables in both experimental and control group.

\section{METHODS}

Design and Setting Quasi experimental control group post test only design was adopted for the study. After obtaining the necessary permission from the concerned authorities and informed consent from the parent of children, the investigator collected data.FLACC scale was used to collect the data. Study was conducted in Jayanagar General Hospital,Bangalore among 60 Preschool children.
The tool consists of 2 sections: Section A It consists of background variables such as children's age, sex, religion, previous experience of IV cannulation and duration of hospital stay.Section B:FLACC Scale was used to assess the level of pain perception among children undergoing IV cannulation. This tool includes five categories of pain behaviors, including facial expression, leg movement, activity, cry and consolability.

Statistical Analysis used Descriptive statistics applied where, data were analyzed by using SPSS software, and Frequency, percentage, tables etc were used to represent the statistical data in the tables and graph and figure. Mann-Whitney U test analysis was used for the significance of post test level of pain.Chi-square test was used to assess the significant association between level of pain with their demographic variable to test the hypothesis.

\section{RESULTS}

Finding of the study showed that in the post test in the experimental group, majority of the subjects $20(66.7 \%)$ had pain score between 5-7(moderate pain) and 10(33.3\%) subject had pain score 8-10(severe pain).In control group, all the subjects $30(100 \%)$ had pain score ( $8-10)$ severe pain. With regard to pain in the experimental group,post mean was 6.73 , standard deviation was 1.08.In the the control group post test ,mean was 9.50, standard deviation was 0.77 . Mann-Whitney test value at $\mathrm{p}<0.05$ was 6.41. Hence,there was exist of statistical significant in the level of pain among hospitalized children undergoing I.V cannulation after flash of light between experimental and control group. In experimental group, there was significance association between the pain score with age, sex, religion,previous experience of I.V cnnulation and duration of hospital stay.In the control group, there was significant association between the pain score with age,gender and religion.There is no significance association between the pain score with previous experience of I.V cannulation and duration of hospital stay. Hence research hypothesis was accepted and null hypothesis was rejected.

\section{CONCLUSION}

The study conclude that In experimental group majority of the subjects $20(66.7 \%)$ had pain score between 5-7(moderate pain) and $10(33.3 \%)$ subject had pain score $8-10$ (severe pain) which was less while comparing to control group.Hence level of 
pain was seen more in control group so using flash of light as a distraction technique during Iv cannulation hwas found to be effective in decreasing the level of pain .

\section{AUTHORS}

First Author - Mrs Pusparani Loitam, Msc Nursing, Padmashree Institute Of Nursing, Bangalore 